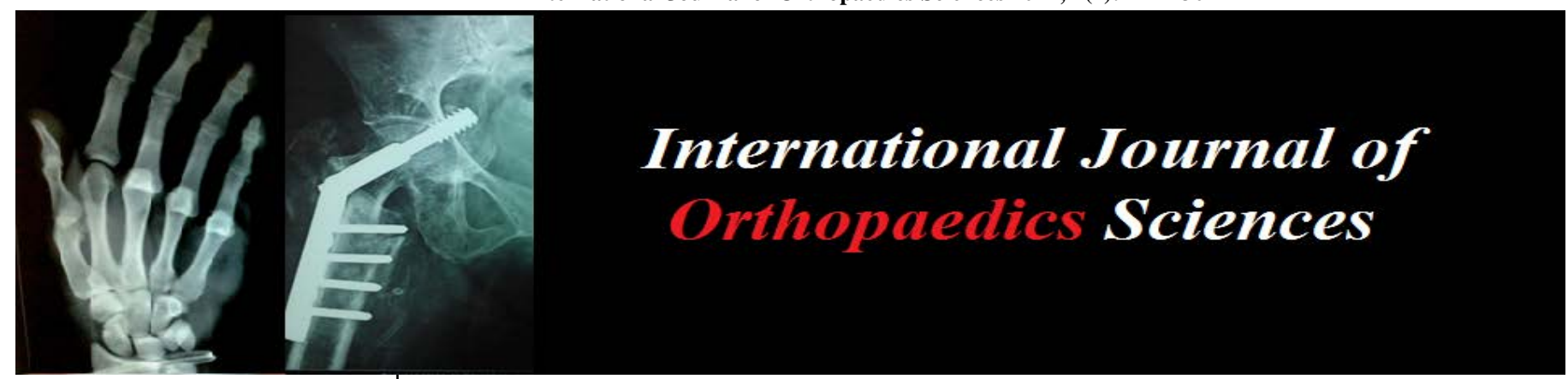

E-ISSN: 2395-1958

P-ISSN: 2706-6630

IJOS 2021; 7(4): 424-430

(C) 2021 IJOS

www.orthopaper.com

Received: 23-07-2021

Accepted: 12-10-2021

Thirunthaiyan MR

Assistant Professor, Department

of Orthopaedics, Sri

Ramachandra Institute of

Higher Education and Research, Chennai, Tamil Nadu, India

Kaustav Mukherjee

Post graduate, Department of

Orthopaedics, Sri Ramachandra

Institute of Higher Education

and Research, Chennai, Tamil

Nadu, India

Tarun Prashanth KR

Assistant Professor, Department of Orthopaedics, Sri

Ramachandra Institute of

Higher Education and Research,

Tamil Nadu, India

Dorai Kumar R

Professor and Unit head,

Department of Orthopaedics, Sri

Ramachandra Institute of

Higher Education and Research,

Chennai, Tamil Nadu, India
Corresponding Author: Kaustav Mukherjee

Post graduate, Department of Orthopaedics, Sri Ramachandra Institute of Higher Education and Research, Chennai, Tamil Nadu, India

\section{Efficacy of intra-articular corticosteroid and hyaluronic acid injections in preventing progression of early osteoarthritis of the knee in Indian population - A single centre prospective study with a follow-up of 18 months}

\author{
Thirunthaiyan MR, Kaustav Mukherjee, Tarun Prashanth KR and Dorai \\ Kumar R
}

DOI: https://doi.org/10.22271/ortho.2021.v7.i4f.2914

\section{Abstract}

Introduction: Osteoarthritis is a degenerative joint disease affecting middle aged to elderly population. Trial of conservative management in form of intra-articular injection with drugs like corticosteroid and hyaluronic acid have been in use for quite some time. We aim to test the efficacy of these drugs when given individually as well in combination in providing pain relief in patients with early-stage osteoarthritis.

Materials and Methods: A prospective study conducted over a period of 4 years (2015-2019) was conducted with a sample size of 150 patients with 50 patients divided equally into 3 groups receiving corticosteroid, hyaluronic acid and combination of both respectively. Patients in age group of $40-65$ years and with Kellgren Lawrence Grade I and II were included in the study. Functional scoring was evaluated pre -procedure, post-procedure at 1 month, and at regular interval of 3 months till 18 months. Visual analogue scale (VAS) and Knee society scoring (KSS) were used to evaluate the results.

Results: Mean age of the patients included in the study was 57.3 years with $59 \%$ female and $41 \%$ male patients. Mean VAS score and mean KSS in corticosteroid group (CS) at 3 months follow-up were 4.92 and 152.81 respectively $(p<0.01)$. For the hyaluronic acid (HA) group, patients had a good relief till 9 months with a mean VAS at 9 months-4.91 and Mean KSS at 9 months-155.26 $(p<0.01)$. However, the combined group showed significantly long pain free period upto 12 months $(p<0.001)$. At end of followup, CS group, HA group and CS with HA group had 12, 8 and 3 patients respectively opting for surgical intervention.

Conclusion: Intra-articular injections provide a good and long period of pain relief in early arthritis. Coinjection of steroids with hyaluronic acid are far superior to any of these drugs administered individually.

Keywords: corticosteroids, hyaluronic acid, osteoarthritis, early

\section{Introduction}

Osteoarthritis (OA) is a clinical syndrome of joint pain with multi-factorial etiopathogenesis that is characterized by gradual loss of articular cartilage, osteophyte formation, subchondral bone remodeling and inflammation of joint ${ }^{[1]}$. It is a chronic progressive joint disease affecting middle aged and elderly population worldwide. Besides cartilage degradation, synovitis, subchondral bone cysts, degeneration of ligaments and menisci, hypertrophy of the joint capsule play an important role in pathogenesis ${ }^{[2]}$.Patients suffering from osteoarthritis have pain and loss of function, leading to disability. It has been the most common form of joint disease and among the top ten causes of disability worldwide ${ }^{[3]}$. Recent studies regarding the epidemiology of osteoarthritis showed an incidence of around $10 \%$ in men and $13 \%$ in women aged 60 years or older, and its incidence is gradually rising ${ }^{[4]}$. A variety of risk factors predisposes an individual to develop osteoarthritis including aging, genetics, trauma, malalignment of knee joint and obesity. OA thus is a major health problem and causing significant financial burden for global economy ${ }^{[5]}$. 
Treatment of osteoarthritis knee is multimodal. With recent developments in field of medicine,there is a wide array of conservative and surgical options to choose for the management of OA knee. Treatment to a large extent is guided by the radiological analysis of the knee joint, as evident on standard Antero-posterior and Lateral view standing $\mathrm{X}$-rays to grade the degree of damage and plan the mode of management. Kellgren -Lawrence (KL) classification of OA knee forms a major basis to decide on the mode of management ${ }^{[6]}$. However, orthopedic surgeons are reluctant to advise operative treatment straight away unless in very advanced disease. Trial of conservative management is advocated in most of the patients including nonpharmacological and pharmacological measures. Lifestyle modifications, weight reduction, exercises, walking supports, bracing, local cooling/heating, acupuncture often do wonders for the patients with very early disease ${ }^{[2]}$. Pharmacological therapies include oral NSAIDs, opioids, collagen supplements. If orally administered drugs also are not giving a pain relief, then only intra-articular injections (corticosteroids, visco-supplement, blood derived products) are offered to the patients as last resort in conservative management. Both corticosteroid and hyaluronic acid (HA) supplementation into the joint via intra-articular injections has been in practice for last 2 decades. There is adequate literature suggesting quick onset of action of corticosteroids, a cost-effective measure, with significant relief of pain in patients which is often shortlived. HA intra-articular injections on other hand though costly gives a comparatively longer pain relief to patients as compared to corticosteroid injections. Though there is ample literature comparing the effectiveness and longevity of these 2 very popular intra-articular injections, hardly there are any literary documentation whether these injections can prevent the disease to progress further and will prevent the patient from landing into advanced arthritis.

We in this study, have aimed to evaluate the progression of the disease in patients who received intra-articular steroid injection (patients with early arthritis - KL grade 1 and KL grade 2). We hypothesized that prompt intra-articular injections in early stages of arthritis can give potential long term pain relief. We followed up the patients for a period of one and half years and noted how many landed up into knee reconstruction surgery on a later date, during our follow-up period.

\section{Materials and Methods}

A prospective study conducted from April 2015 to December 2019 was designed with the study conducted at our institute. A total of 150 patients were included in the study, who were divided into 3 groups based on the intra-articular injection content - receiving only corticosteroid injection, only hyaluronic acid injection and lastly the group who received combination of both corticosteroid and hyaluronic acid(either on the same sitting or later on). Patients without significant relief even after the injections, were managed surgically. All patients were evaluated radiologically by standard AP and Lateral view X-rays of knee joint before the procedure.

Inclusion criteria were - Age $>40$ years but $<65$ years, knees with Grade I/Grade II on Kellgren and Lawrence classification (Figure 1), no prior deformities of knee joint, no history of fractures, patients dis-satisfied with pain control with analgesics and oral supplementations. Patients with advanced arthritis, involvement of more than 2 compartments of knee joint, bilateral osteoarthritis knee, current infection in limb, previous history of trauma/surgeries, history of crystalline arthropathy or inflammatory arthritis or neuropathic arthropathy, patients lost to follow-up were excluded from the study. Patients requiring more than 2 intraarticular injections in the same knee joint were also excluded from the study.

\begin{tabular}{|ll|}
\hline Grade & Radiologic Findings \\
\hline I & Doubtful narrowing of joint space and possible osteophytic \\
II & lipping \\
III & $\begin{array}{l}\text { Definite osteophytes and possible narrowing of joint space } \\
\text { space, some sclerosis and possible deformity of bone }\end{array}$ \\
IV & $\begin{array}{l}\text { contour } \\
\text { Large osteophytes, marked narrowing of joint space, severe } \\
\text { sclerosis and definite deformity of bone contour }\end{array}$ \\
\hline
\end{tabular}

Fig 1: Kellgren Lawrence radiological classification of osteoarthritis of knee joint

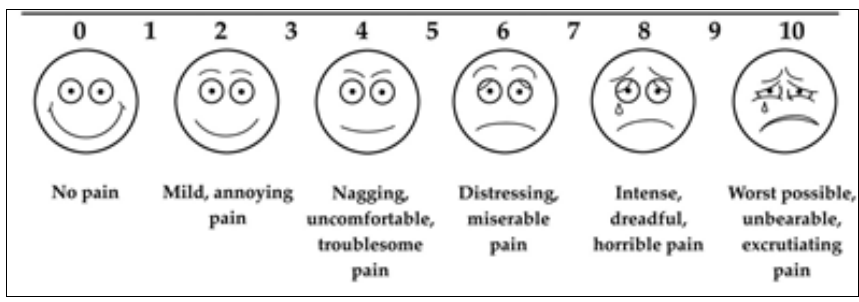

Fig 2: Visual analogue scale

Table 1: Knee society scoring system

\begin{tabular}{|c|c|c|c|}
\hline Parameters & Subgroups & Variables & Points \\
\hline \multirow{7}{*}{ Pain } & & None & 50 \\
\hline & & Mild/occasional & 45 \\
\hline & & Mild, stairs only & 40 \\
\hline & & Mild, walking \& stairs & 30 \\
\hline & & Moderate,occasional & 20 \\
\hline & & Moderate, continual & 10 \\
\hline & & Severe & 0 \\
\hline \multirow{6}{*}{ Stability } & & $<5 \mathrm{~mm}$ & 10 \\
\hline & & $5-10 \mathrm{~mm}$ & 5 \\
\hline & Antero-posterior & $>10 \mathrm{~mm}$ & 0 \\
\hline & Medio-Lateral & $<5$ degrees & 15 \\
\hline & & 6-9 degrees & 10 \\
\hline & & 10-14 degrees & 0 \\
\hline Range of motion & Every 5 degree $=1$ point (maximum of 25 points) & & \\
\hline \multirow{4}{*}{ Function } & Walking & Unlimited & 50 \\
\hline & & $>10$ blocks & 40 \\
\hline & & 5-10 blocks & 30 \\
\hline & & $<5$ blocks & 20 \\
\hline
\end{tabular}




\begin{tabular}{|c|c|c|c|}
\hline & Stairs & $\begin{array}{c}\text { House bound } \\
\text { Unable } \\
\text { Normal up and down } \\
\text { Up and down with rail } \\
\text { Up with rail, unable down } \\
\text { Unable }\end{array}$ & $\begin{array}{c}10 \\
0 \\
50 \\
40 \\
15 \\
0\end{array}$ \\
\hline Deductions & $\begin{array}{l}\text { Flexion contracture } \\
\text { Extension Lag } \\
\text { Functional scoring }\end{array}$ & $\begin{array}{c}\text { 5-10 degrees } \\
11-15 \text { degrees } \\
16-20 \text { degrees } \\
>20 \text { degrees } \\
<10 \text { degrees } \\
10-20 \text { degrees } \\
>20 \text { degrees } \\
\text { Cane or stick } \\
2 \text { cane/Walking stick } \\
\text { Crutches/walker }\end{array}$ & $\begin{array}{c}2 \\
5 \\
10 \\
15 \\
5 \\
10 \\
15 \\
5 \\
10 \\
20\end{array}$ \\
\hline
\end{tabular}

Prior to procedure, patients were evaluated by proper history taking, clinically and by blood investigations (Complete blood counts, Erythrocyte sedimentation rate, C-Reactive proteins, RA factor, and Serum uric acid). Procedures were done on an outpatient basis under sterile conditions and precautions. Entry to the lateral joint space (Figure 3) was made with 18G needle after proper draping and then injected with corticosteroid (Methylprednisolone) or hyaluronic acid (Hyalgan) (Figure 4). Pre and post-injection evaluation of pain (at 1 month) was done using the Visual analogue scale (VAS) and Knee society score (KSS). Serial evaluation was done at every 3 months for next 18 months, and patients were followed up closely during this period (Figure 2, Table 1). Statistical analysis was done using SPSS 19(IBM Corp., USA). All data are presented as mean+/- standard deviation in tables. VAS score and KSS were evaluated using independent Student's t test.
Comparison in each of the 3 groups was compared using Student's t tests. $P<0.05$ was considered significant.

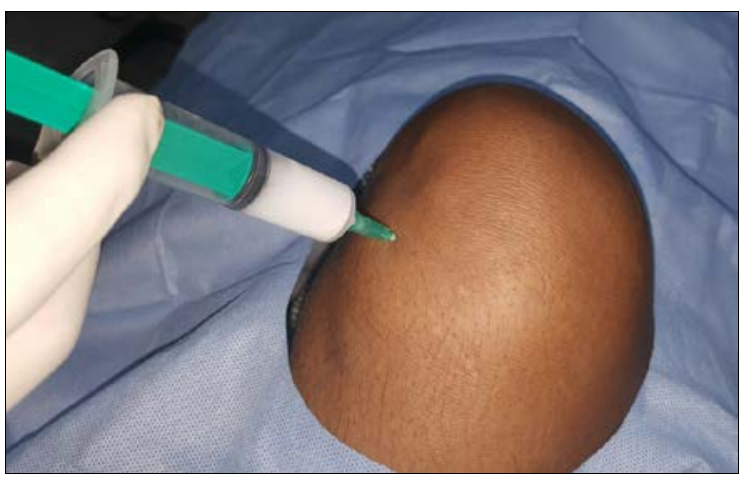

Fig 3: Corticosteroid intra-articular injection into the knee joint through lateral joint entry
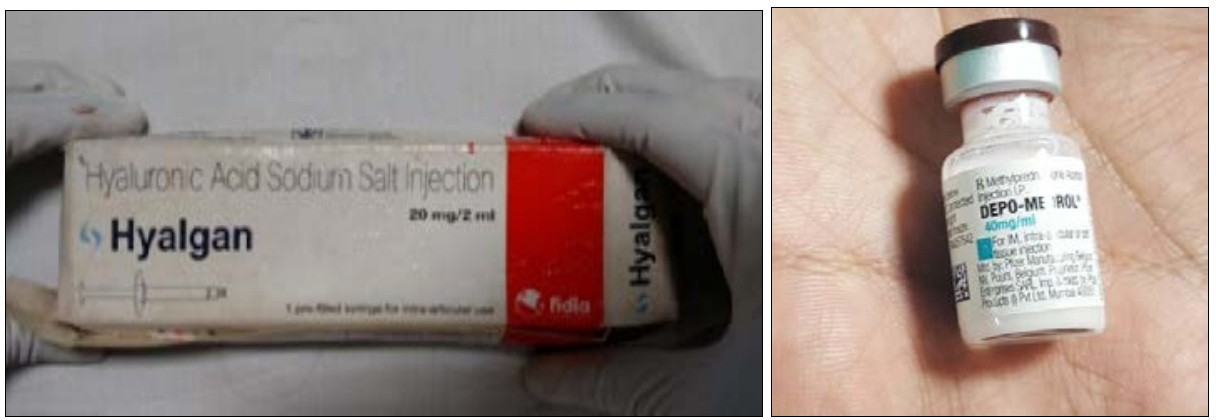

Fig 4: Illustrates the hyaluronic acid and corticosteroid injection used in the procedures

\section{Results}

\subsection{Demographics}

A total of 150 patients were included in the study out of the 389 patients initially included as majority were lost to followup. Equal numbers of cases were chosen in all the 3 groups with 50 cases each in corticosteroid (CS), hyaluronic acid (HA) and combination of CS with HA groups. We had patients with age ranging from 46 years to 65 years, with mean age of 57.3 years. Out of this total study group, 89 were female and 61 were male patients. 84 patients had intra- articular injections done on their right knee while 66 patients to their left knee. Group 1 (receiving CS intra-articular injections only) had mean age of 55.5 yrs with a mean BMI of $25.1 \mathrm{~kg} / \mathrm{m}^{2}$, Group 2 (receiving hyaluronic acid intra-articular injection only) had a mean age of 57 yrs with a mean BMI of $26.4 \mathrm{~kg} / \mathrm{m}^{2}$ and lastly the group including administration of corticosteroid with hyaluronic acid together included patients with mean age of 59.6 yrs with a mean BMI of $27.9 \mathrm{~kg} / \mathrm{m}^{2}$ (Table 2).

Table 2: Demographic representation of patient distribution in three different study groups

\begin{tabular}{|c|c|c|c|}
\hline & Group I (CS) & Grade II (HA) & Group III (CS+HA) \\
\hline \multirow{2}{*}{ Sex distribution } & Males-20 & Males-24 & Males-17 \\
\cline { 2 - 4 } & Females-30 & Females-26 & Females-33 \\
\hline \multirow{2}{*}{ Side } & Right-32 & Right-22 & Right-30 \\
\cline { 2 - 4 } & Left-18 & Left-28 & Left-20 \\
\hline Mean age (years) & $55.5+/-1.5$ & $57.1+/-1.2$ & $59.6+/-1.1$ \\
\hline Mean BMI $\left(\mathrm{kg} / \mathrm{m}^{2}\right)$ & $25.1+/-1.1$ & $26.4+/-0.9$ & $27.9+/-0.8$ \\
\hline
\end{tabular}




\subsection{Analysis of functional outcomes}

Visual analogue score (VAS) was utilized to plot the functional outcome of the 3 groups of patients. Scale of range 0-10 with no pain graded 0 , mild pain 1-3, 4-6 graded as moderate pain, 7-9 as severe pain and 10 as worst pain possible. VAS was recorded pre and post procedure(at 1 month) and at 3 months, 6 months, 9 months, 12 months, 15 months and 18months respectively for all the groups. The mean VAS of CS group, HA group and CS with HA group were $6.51,6.68$ and 6.89 respectively at initial presentation
(Table 3). Post procedure VAS improved and showed a steady decline for the first 3months. Follow-up at 3 months showed corticosteroid group mean VAS score to increase from 3.08 to 4.02 and rise steadily thereafter on subsequent follow-ups. Mean VAS score for HA group increased at 9 months followup from 4.8 to 4.91. However, the combined CS with HA group showed a increase in mean VAS score only at 12 months follow-up from3.62 to 3.91, only to rise steadily further from there on. (Figure 5).

Table 3: Visual analogue scoring (VAS) of the 3 groups and their follow-ups at 3 months interval

\begin{tabular}{|c|c|c|c|c|}
\hline & Group & $\mathbf{N}$ & Mean & S.D. \\
\hline \multirow{3}{*}{ Pre-procedure VAS } & $\mathrm{CS}$ & 50 & 6.51 & 1.005 \\
\hline & HA & 50 & 6.68 & 0.928 \\
\hline & $\mathrm{CS}+\mathrm{HA}$ & 50 & 6.89 & 1.002 \\
\hline \multirow{3}{*}{ Post-procedure VAS at 1 month } & $\mathrm{CS}$ & 50 & 3.08 & 1.192 \\
\hline & HA & 50 & 5.18 & 0.962 \\
\hline & $\mathrm{CS}+\mathrm{HA}$ & 50 & 4.06 & 0.983 \\
\hline \multirow{3}{*}{ VAS at 3 months } & CS & 49 & 4.02 & 1.195 \\
\hline & HA & 50 & 4.96 & 1.046 \\
\hline & $\mathrm{CS}+\mathrm{HA}$ & 50 & 4.01 & 1.013 \\
\hline \multirow{3}{*}{ VAS at6months } & CS & 46 & 4.92 & 1.301 \\
\hline & HA & 48 & 4.80 & 1.129 \\
\hline & $\mathrm{CS}+\mathrm{HA}$ & 50 & 3.98 & 0.976 \\
\hline \multirow{3}{*}{ VAS at 9 months } & $\mathrm{CS}$ & 41 & 5.21 & 1.215 \\
\hline & HA & 46 & 4.91 & 1.113 \\
\hline & $\mathrm{CS}+\mathrm{HA}$ & 49 & 3.62 & 1.017 \\
\hline \multirow{3}{*}{ VAS at 12 months } & $\mathrm{CS}$ & 40 & 5.42 & 1.136 \\
\hline & HA & 43 & 5.01 & 1.017 \\
\hline & $\mathrm{CS}+\mathrm{HA}$ & 47 & 3.91 & 0.968 \\
\hline \multirow{3}{*}{ VAS at 15 months } & $\mathrm{CS}$ & 39 & 5.82 & 1.118 \\
\hline & HA & 43 & 5.56 & 1.107 \\
\hline & $\mathrm{CS}+\mathrm{HA}$ & 47 & 4.31 & 1.008 \\
\hline \multirow{3}{*}{ VAS at 18 months } & $\mathrm{CS}$ & 38 & 6.21 & 1.138 \\
\hline & HA & 42 & 6.32 & 1.117 \\
\hline & $\mathrm{CS}+\mathrm{HA}$ & 47 & 5.83 & 0.985 \\
\hline (S.D- standard deviation) & & & & \\
\hline
\end{tabular}

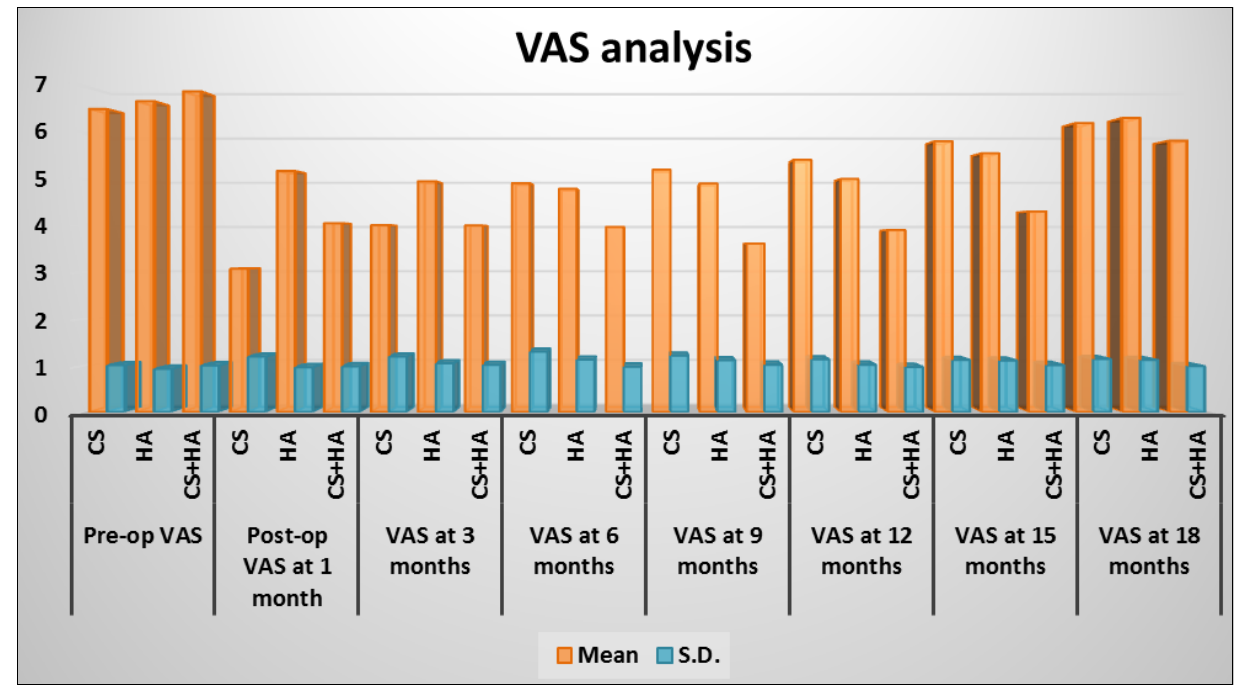

Fig 5: VAS analysis of all three groups

Knee society scoring (KSS) template was used to record individual scoring of the patients in 3 groups at pre-procedure and at regular follow-up visits post-procedure. Mean KSS recorded at initial visits in 3 groups were 140.58, 140.80 and 141.86 respectively (Table 4). Post-injection and follow-up at 1 month all the 3 groups showed good improvement in scores with KSS scoring being 155.34, 150.30, 152.31 respectively for the 3 groups. Regular follow-ups were done and scoring were recorded showing a decline in KSS for the corticosteroid and hyaluronic acid group at 6 months follow-up recording 152.81 and 158.32 from their previous scores of 155.84 and 160.30 respectively. Combined corticosteroid and hyaluronic acid group showed decline at 9 months follow-up recording a KSS of 159.21 from its previous score of 160.86 (Figure 6). 
Table 4: Knee society scoring (KSS) for patients in 3 groups and follow up at regular intervals

\begin{tabular}{|c|c|c|c|c|}
\hline & Group & $\mathbf{N}$ & Mean & S.D. \\
\hline \multirow{3}{*}{ Pre-procedure KSS } & $\mathrm{CS}$ & 50 & 140.58 & 12.360 \\
\hline & HA & 50 & 140.80 & 9.223 \\
\hline & $\mathrm{CS}+\mathrm{HA}$ & 50 & 141.86 & 8.619 \\
\hline \multirow{3}{*}{ Post-procedure KSS at 1 month } & CS & 50 & 155.34 & 10.464 \\
\hline & HA & 50 & 150.30 & 8.830 \\
\hline & $\mathrm{CS}+\mathrm{HA}$ & 50 & 152.31 & 8.916 \\
\hline \multirow{3}{*}{ KSS at 3 months } & $\mathrm{CS}$ & 49 & 155.84 & 9.561 \\
\hline & HA & 50 & 160.30 & 8.911 \\
\hline & $\mathrm{CS}+\mathrm{HA}$ & 50 & 158.31 & 8.689 \\
\hline \multirow{3}{*}{ KSS at 6 months } & CS & 46 & 152.81 & 10.116 \\
\hline & HA & 48 & 158.32 & 10.102 \\
\hline & $\mathrm{CS}+\mathrm{HA}$ & 50 & 160.86 & 9.146 \\
\hline \multirow{3}{*}{ KSS at 9 months } & CS & 41 & 150.70 & 9.917 \\
\hline & HA & 46 & 155.26 & 9.315 \\
\hline & $\mathrm{CS}+\mathrm{HA}$ & 49 & 159.81 & 8.615 \\
\hline \multirow{3}{*}{ KSS at 12 months } & CS & 40 & 148.71 & 8.515 \\
\hline & HA & 43 & 150.56 & 7.417 \\
\hline & $\mathrm{CS}+\mathrm{HA}$ & 47 & 155.80 & 7.516 \\
\hline \multirow{3}{*}{ KSS at 15 months } & $\mathrm{CS}$ & 39 & 145.61 & 7.117 \\
\hline & HA & 43 & 149.17 & 7.105 \\
\hline & $\mathrm{CS}+\mathrm{HA}$ & 47 & 152.75 & 6.917 \\
\hline \multirow{3}{*}{$\begin{array}{c}\text { KSS at } 18 \text { months (S.D- Standard } \\
\text { Deviation) }\end{array}$} & CS & 38 & 141.17 & 7.321 \\
\hline & HA & 42 & 145.79 & 7.567 \\
\hline & $\mathrm{CS}+\mathrm{HA}$ & 47 & 150.49 & 5.584 \\
\hline
\end{tabular}

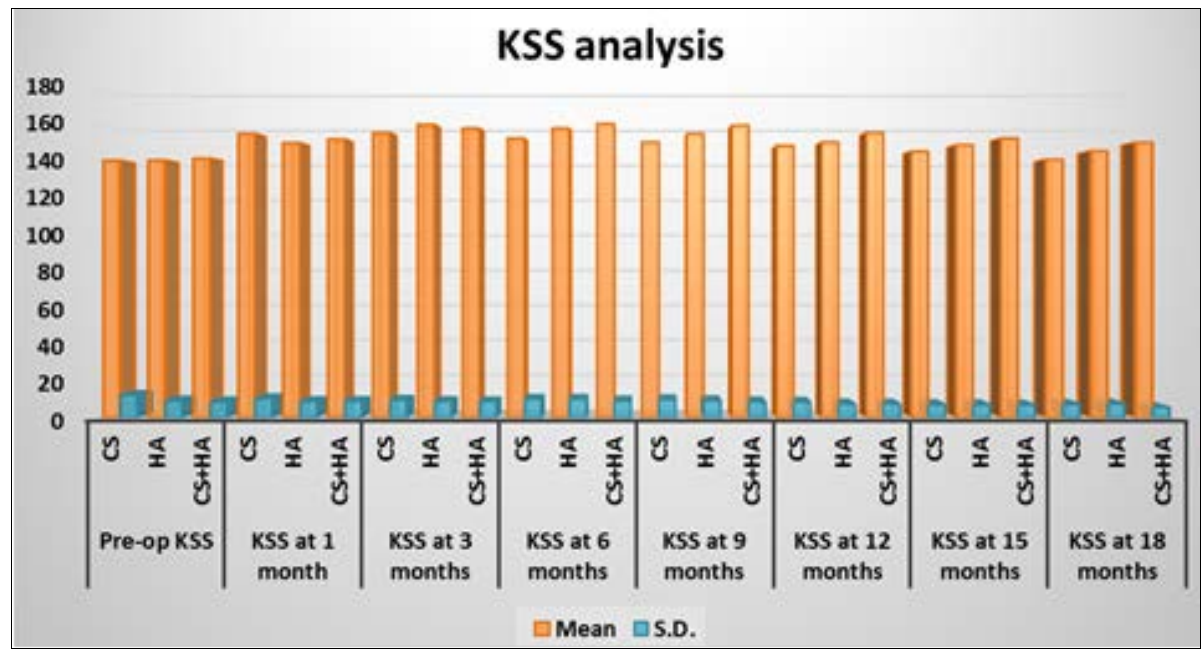

Fig 6: KSS analysis of all three groups

\subsection{Analysis of the affectivity of the intra articular steroid injections}

The 3 group of patients were followed up for the requirement of surgical procedures and the time to intervention was noted. Group 1 had 12 patients undergoing surgical procedure due to inadequate pain control. Mean time to surgical intervention was 22.8 weeks from the time of steroid administration. Group 2 had 8 patients who underwent surgical interventions with a mean time duration from hyaluronic acid to surgical procedure being 28.1 weeks. Three patients from group 3 opted for surgical reconstructive procedure at a mean time of 49.5 weeks (Table 5).

Table 5: Depicts the cases from each group undergoing operative procedures

\begin{tabular}{|c|c|c|c|}
\hline & Group I & Group II & Group III \\
\hline Number of cases undergoing surgical intervention & 12 & 8 & 3 \\
\hline Type of surgeries done & UKR-1, HTO-2,HFO-4,TKR-8 & HFO-3, TKR-5 & TKR-3 \\
\hline Mean time to intervention from injection & 32.8 weeks & 38.1 weeks & 49.5 weeks \\
\hline Mean Age of patients undergoing surgeries & 61.2 years & 62.9 years & 63.2 years \\
\hline Average BMI & 28.5 & 29.9 & 28.7 \\
\hline
\end{tabular}

(UKR- unicompartmental knee replacement, HTO- high tibial osteotomy, HFO- high fibular osteotomy, TKR- total knee replacement)

\section{Discussion}

Osteoarthritis of knee joint is a chronic disabling disease associated with pain and deformities posing a great challenge in carrying out day to day daily activities. The primary goal in treatment remains pain reduction, improvement of joint mobility and function. Decreasing the progression of the 
disease remains an important secondary goal [7]. Oral pharmacological treatment may be effective initially, but a meta-analysis done by Bannuru et al. ${ }^{[8]}$ in 2015 suggested oral NSAIDs as inferior to intra-articular injections in management of osteoarthritis.

Intra -articular steroid or hyaluronic acid injections have been in use for quite some time. Both these have given significant relief to patients on immediate basis but their efficacy in providing good pain relief in long run has been in question. However, accurate intra-articular injection into the knee joint is mandatory to see desired results. Jones et al. ${ }^{[9]}$ addressed the issue by obtaining a single plain joint radiograph after joint aspiration and injecting a radio-opaque dye along with corticosteroid. Accurate injection was also associated with successful aspiration of synovial fluid at time of injection. In our study, we also followed the same protocol of aspirating the synovial fluid before injecting the desired drug.

C Tate Hepper et al. ${ }^{[10]}$ in the systemic review about the efficacy and duration of intra-articular corticosteroid injection for knee osteoarthritis mentioned a significant pain relief at 1 week with triamcinolone showing better results than any other steroids.Another review article by $\mathrm{N}$ Bellamy et al. ${ }^{[11]}$ on intra-articular corticosteroid treatment in osteoarthritis of knee, which showed significant pain reduction between 2 weeks to 3 weeks but the effect wearing off, was noted at around 8 weeks to 12 weeks. A comparison study was done with hyaluronic acid group but no statistically significant difference was found with those receiving corticosteroid at 1 to 4 weeks post-injection. However, a statistically significant difference was noted at 5 to 13 weeks post-injection. No difference in efficacy was noted at 45 to 52 weeks in these two groups though. In our study, the corticosteroid group (injected with methylprednisolone) and the hyaluronic acid group showed significant pain reduction at 1 month post procedure as measured on VAS and KSS. However, there was a statistically significant difference at 12 weeks(3 months) between the CS and HA group, with HA group faring better in terms of pain and functional scoring than the CS $\operatorname{group}(p<$ 0.01). Leardini et al. ${ }^{[12]}$ in his randomized controlled trial stated that HA was superior to CS in terms of duration of pain relief. A.Sawara et al. ${ }^{[13]}$ in another randomized controlled trial stated that a single dose of high viscosity HA shows superior range of motion and pain relief as well as improvements in clinical results as compared to CS. We had a similar consensus in our study with HA group having a longer pain free period (24 weeks) as compared to CS group.

Intra-articular single shot co-injection of hyaluronic acid and corticosteroid is a recent go to method of treatment of osteoarthritis of knee due to their longer period of pain relief. Shan-Zhen Wang et al. ${ }^{[14]}$ in their study compared the effectivity between combined HA with CS injection and HA alone group, including 120 patients with osteoarthritis (included all patients from KL grade II to IV). The coinjection group showed better pain relief and functional scores at 1 week, 1 month, 3 months and 6 months. However results were not statistically significant beyond 6 months. In our study, we included only patients with KL grade I and II, who showed statistically significant difference in pain relief and functional outcome upto 9 months as compared to other 2 groups $(p<0.001)$.

We, in our study, have followed up the patients for a period of 18 months, which is a quite a long time, where in most of the literature have studies up to 6 months follow-up. All our patients were early stage osteoarthritis and hence these drugs provided longer relief as compared to other studies. On completing the follow-up for 18 months, patients undergoing surgical intervention were noted and evaluated. Twelve patients from CS group, 8 patients from HA group and only 3 patients from CS with HA group required surgery. The mean time to surgical intervention in the co-injection group was 49.5 weeks as compared to relatively early surgical interventions in the other two groups. Thus, clearly showing that the co-injection group had better functional outcomes and providing a longer time before the patient needed a surgical procedure for better pain relief.

We list some limitations in our study. Only one dose of injection was administered to all the study groups. None of the patients were given a re-injection when the patients came back with complains of pain. Patients coming back with poor control of pain after the first dose of injection were counseled for surgery directly and in a way many were lost to follow-up. Secondly, all these patients receiving intra-articular injections were also on other modalities of physical treatment like lifestyle modifications, exercises, physiotherapy, oral NSAIDs, oral collagen supplementations. In such a scenario getting to know the efficacy of these agents alone and its role in pain control is difficult to assess. Lastly, only patients with early arthritis were included in the study (Kellgren Lawrence Grade I and II). The efficacy of these drugs in pain control in advanced arthritis could not be evaluated.

\section{Conclusion}

Intra-articular injections are far better agents in providing good pain relief than oral therapies in patients opting for conservative management of osteoarthritis of knee. Early diagnosis and early intervention is the key with most patients doing well for a relatively longer time. Co-injection of hyaluronic acid with corticosteroid is far more superior than any of these agents individually administered. All these should be clubbed with good physical therapy and lifestyle modifications with exercises. If intervened early, intraarticular injections can give longer periods of pain relief than when it is used in advanced arthritis, with intra-articular steroids giving relief up to 3 months, hyaluronic acid injection up to 9 months and co-injections giving symptomatic relief up to 12 months. Although no evidence of alteration in pathogenesis of the disease has been seen with these agents, but they definitely help to slow down the wear and tear of the joint when administered in early stage of arthritis giving a good pain relief for a longer time and a reasonably pain free knee for time to come.

Acknowledgement - We acknowledge the help of Dr. Indrakshi Basak and Dr. Ashik Mohammed in tabulating the data and helping with statistical analysis.

\section{Funding- nil}

Conflict of interest- nil

\section{References}

1. Martel-Pelletier J, Boileau C, Pelletier JP, Roughley PJ. Cartilage in normal and osteoarthritis conditions. Best practice \& research Clinical rheumatology 2008;22(2):351-84.

2. Ayhan E, Kesmezacar H, Akgun I. Intraarticular injections (corticosteroid, hyaluronic acid, platelet rich plasma) for the knee osteoarthritis. World journal of orthopedics 2014;5(3):351.

3. National Collaborating Centre for Chronic Conditions (Great Britain), National Institute for Clinical Excellence 
(Great Britain). Osteoarthritis: national clinical guidelines for care and management in adults. Royal College of Physicians.

4. Zhang Y, Jordan JM. Epidemiology of osteoarthritis. Clinics in geriatric medicine 2010;26(3):355-69.

5. Neogi T. The epidemiology and impact of pain in osteoarthritis. Osteoarthritis and cartilage. 2013;21(9):1145-53.

6. Petersson IF, Boegård T, Saxne T, Silman AJ, Svensson B. Radiographic osteoarthritis of the knee classified by the Ahlbäck and Kellgren\& Lawrence systems for the tibiofemoral joint in people aged 35-54 years with chronic knee pain. Annals of the rheumatic diseases 1997;56(8):493-6.

7. Askari A, Gholami T, NaghiZadeh MM, Farjam M, Kouhpayeh SA, Shahabfard Z. Hyaluronic acid compared with corticosteroid injections for the treatment of osteoarthritis of the knee: a randomized control trail. Springerplus 2016;5(1):1-6.

8. Bannuru RR, Schmid CH, Kent DM, Vaysbrot EE, Wong JB, McAlindon TE. Comparative effectiveness of pharmacologic interventions for knee osteoarthritis: a systematic review and network meta-analysis. Annals of internal medicine2015;162(1):46-54.

9. Jones A, Doherty M. Intra-articular corticosteroids are effective in osteoarthritis but there are no clinical predictors of response. Annals of the rheumatic diseases 1996;55(11):829-32.

10. Hepper CT, Halvorson JJ, Duncan ST, Gregory AJ, Dunn WR, Spindler KP. The efficacy and duration of intraarticular corticosteroid injection for knee osteoarthritis: a systematic review of level I studies. JAAOS-Journal of the American Academy of Orthopaedic Surgeons 2009;17(10):638-46.

11. Bellamy N, Campbell J, Welch V, Gee TL, Bourne R, Wells GA. Intraarticular corticosteroid for treatment of osteoarthritis of the knee. Cochrane Database of Systematic Reviews, 2006, (2).

12. Leardini G, Mattara L, Franceschini M, Perbellini A. Intra-articular treatment of knee osteoarthritis. A comparative study between hyaluronic acid and 6-methyl prednisolone acetate. Clinical and experimental rheumatology 1991;9(4):375-81.

13. Skwara A, Ponelis R, Tibesku CO, Rosenbaum D, FuchsWinkelmann S. Gait patterns after intraarticular treatment of patients with osteoarthritis of the knee-hyaluronan versus triamcinolone: a prospective, randomized, doubleblind, monocentric study. European journal of medical research 2009;14(4):157-64.

14. Wang SZ, Wu DY, Chang Q, Guo YD, Wang C, Fan WM. Intra-articular, single-shot co-injection of hyaluronic acid and corticosteroids in knee osteoarthritis: A randomized controlled trial. Experimental and therapeutic medicine 2018;16(3):1928-34. 